\title{
(2) Nuclear Magnetic Resonance
}

\author{
Noboru ARIMIZU \\ School of Medicine, Department of Radiology Chiba University
}

The clinical application of Nuclear Magnetic Resonance (NMR) is exclusive in forming tomographic images of the body from the outside at present. In near future, however, NMR spectrometry, having developed in the field of analytical chemistry and biochemistry, will be important means of clinical examinations for cancer when MRS of the target volume alone can be easily measured from the outside.

The study purposes to surmarize the current state of NMR imaging (MRI) for diagnosis of cancer. In present Japan, approximate two hundred facilities use MRI for clinical purposes and half of them utilize homemade MRI machines, mainly equipping resistive magnets.

The relaxation time of NMR, composed of T-1 or $\mathrm{T}-2$, is essential of producing image contrast on MRI. And, relaxation time, dependent on free water content in tissues, frequently increases in malignat conditions, making useful image contrast from the surroundings. In General, MRI is superior in image contrast of soft tissues to X-ray computed tomography (CT).

Blood flow in vessels do not originate MRI image signals, bringing useful informations for identifying blood vessels from the surrounding soft tissues without contrast media.

Time needed for taking a picture ranges from two to twenty minutes in ordinary MRI and breath-hold is impossible during them. The breathing indispensably causes small movements in organs or tissues of the chest and upper abdomen, frequently deteriorating sharpness of images as well as their contrast. These often make difficult to find out a small lesion of low contrast. Latest researches are directed to minimize time for taking a picture.

Enhancement of image contrast offers potentials of promoting diagnostic usefulness of MRI. Contrast agents in MRI are now on progress with clinical trials, and Gadopentic acid (Ga-DTPA) has been examined by twelve institutes in Japan. Contrast enhancement is attainable on T-1 weight images, disclosing its usefulness in discriminating the extent of tumor from edema and other surroundings. These are illustrated by images of mass lesins in the spinal cord and brain stem.

\section{(3) Immunodetection of Small Cell Lung Cancer}

\section{Tetsuro OKABE}

The Third Department of Internal Medicine, Faculty of Medicine, University of Tokyo, Tokyo

For diagnosis and treatment of small cell lung cancer (SCLC), we have developed four monoclonal antibodies ${ }^{1)}$, one of which is useful for specific diagnosis of $\mathrm{SCLC}^{2}$. The monoclonal antibody designated as TFS-4 is found to be highly specific for $\mathrm{SCLC}^{3)}$. It reacts with SCLC but unreactive with squamous cell carcinoma, adenocarcinoma, or large cell carcinoma of the lung. It can distinguish SCLC from non-SCLC in histologic diagnosis at transbronchial biopsy, and in cytological identification of tumor cells in malignant effusions and inflitrated bone marrow ${ }^{4}$. Radiolabeled TFS-4 specificially accumulated into SCLC tumors transplanted into nude mice and inhibited the growth of the tumors in vivo. In this paper, we described the specific diagnosis and treatment 
of SCLC xenografts in nude mice by TFS-4 monoclonal antibody.

Immunohistochemistry: Reactivity of TFS-4 with surgical materials of primary lung cancers is shown in Table 1. All of the SCLC tumors showed positive reactions. TFS-4 was reactive with none of the conventional squamous cell carcinomas, nor large cell carcinomas of the lung. In the positive cases the reaction appeared to be confined to cell surface. Reactivity of TFS-4 with cancers from other organs is also shown in Table 1. None of the colorectal cancers, gastric cancers, or lymphomas was reactive with TFS-4. Interestingly, carcinoid tumors and a neuroblastoma showed positive reactions.

In normal tissues, most remarkable staining was demonstrated on central nervous tissues and neuroendocrine cells, including the adrenal gland (medulla, and zona glomerulosa), glandular cells of the thyroid, Leydig cells of the testis, and stromal cells of the ovary. (Table 2). In peripheral nerves, Schwann cells and nerve endings were weakly stained. No reactivity was detected with other tissues including trachea, lung, liver, kidney, colon, pancreas, skin, striated muscle, spleen, bone marrow, or peripheral blood cells. The islet of the pancreas, enteroendocrine cells, and Kulchitski cells of the lung were found to be unreactive. An unexpected finding was that the antibody was reactive with cell membranes of cardiac muscles and some smooth muscle cells (alimentary tract and myometrium of the uterus).Gamma Scintigraphy of ${ }^{131}$ I-labeled TFS-4: Twenty-four hours after the injection of ${ }^{131}$ I-labeled TFS-4, gamma scitigraphy showed whole mouse figures and no tumor figures. On the 4th day, the tumor was clearly imaged. After seven days, background radioactivity decreased and tumor figures were more clearly demarcated (Fig. 1). The gamma scintigrams of all five nude mice with SCLC xenografts showed a similar time course of tumor imaging. The thyroid glands are not suppressed by excess iodine and free ${ }^{131} \mathrm{I}$ may be taken up in the thyroid glands (Fig. 1).

TFS-4 Distribution in Tumors and Visceral Organs: The ${ }^{131} \mathrm{I}$ distribution in the nude mice with SCLC xenograft was examined after seven day of the ${ }^{131}$ I-TFS-4 injection. Figure 2 shows
Table 1. Reactivity of TFS-4 with primary lung cancers and other malignancies.

Positivity was arbitrarily designated as the staining of more than $10 \%$ of malignant cells.

\begin{tabular}{lc}
\hline Histological diagnosis & No. positive/no. tested \\
\hline Primary lung cancers & \\
Small-cell lung cancer & $15 / 15$ \\
Squamous cell carcinoma & $0 / 20$ \\
Adenocarcinoma & $0 / 20$ \\
Large-cell lung cancer & $0 / 2$ \\
Carcinoid tumor & $2 / 2$ \\
Other malignancies & \\
Colorectal cancer & $0 / 17$ \\
Gastric cancer & $0 / 15$ \\
Breast cancer & $0 / 1$ \\
Lymphoma & $0 / 20$ \\
Thymoma & $0 / 1$ \\
Leiomyosarcoma & $0 / 1$ \\
Meningioma & $0 / 5$ \\
Neuroblastoma & $1 / 1$ \\
\hline
\end{tabular}

Table 2. Reactivity of TFS-4 with normal tissues Cell types with positive reactions were shown in parentheses.

\begin{tabular}{ll}
\hline \multicolumn{1}{c}{ Positive tissues } & \multicolumn{1}{c}{ Negative tissues } \\
\hline Central nervous tissues & Trachea \\
Cerebrum & Lung \\
Cerebellum & Liver \\
Spinal cord & Kidney \\
Peripheral nerves & Colon \\
Adrenal gland (medulla and zona & Peripheral blood cells \\
$\quad$ glomerulosa) & Bone marrow \\
Thyroid (glandular cells) & Striated muscles \\
Testis (Leydig cells) & Kulchitski cells of the lung \\
Ovary (stromal cells) & Enteroendocrine cells \\
Cardiac muscles & Islet cells of the pancreas \\
Smooth muscles (alimentary tract, & \\
$\quad$ myometrium) & \\
\hline
\end{tabular}

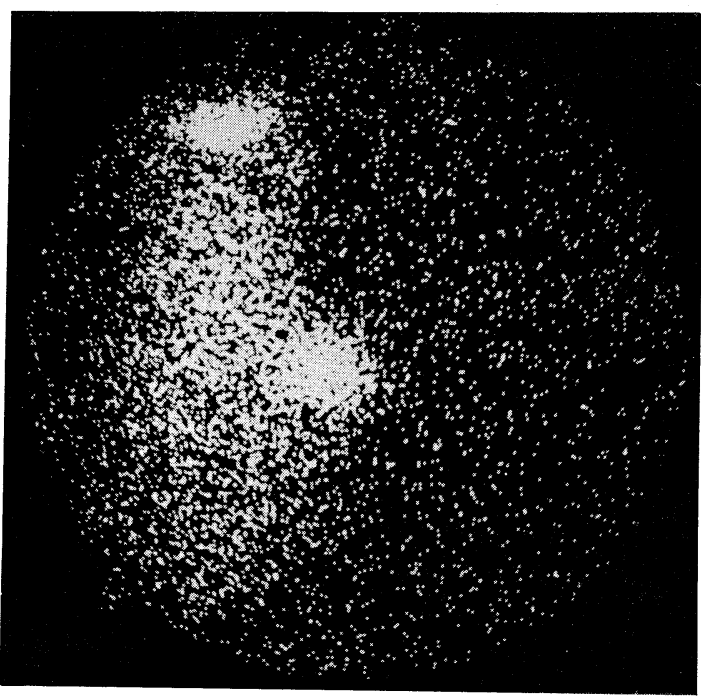

Fig. 1 A gamma scintigram of a nude mouse with a SCLC xenograft in rifgt ' flank, which was injected with radiolabelled TFS-4.

the distribution of ${ }^{131} \mathrm{I}$ which gives counts per minutes (cpm) value (per mg tissue) of each tumor 


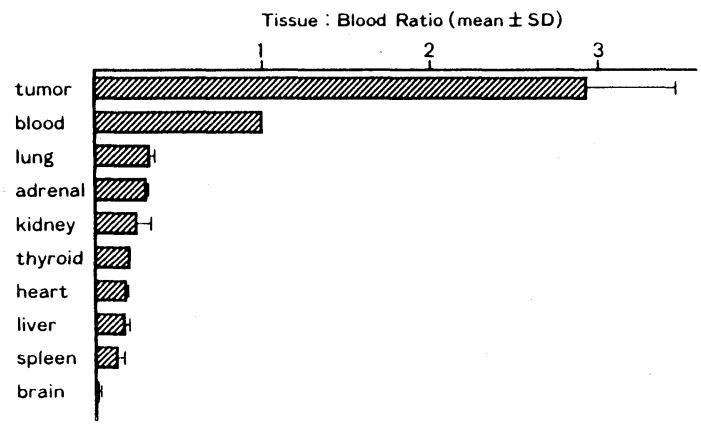

Fig. 2. Distribution of radiolabeled TFS-4 in nude mice with SCLC xenografts.

and organ. The tumors showed specific accumulation of TFS-4 monoclonal antibody seven days after injection. Little radioactivity was detected in the brain tissues, which may be due to bloodbrain barrier.

Treatment of SCLC Xenografts by ${ }^{131}$ I-TFS-4: When the ${ }^{131}$-labeled TFS was injected into nude mice with SCLC xenografts, the growth of the tumor was strongly inhibited. However, the control monoclonal antibody labeled with ${ }^{131}$ I showed no significant effects on the growth of SCLC xenografts.

These observations suggest that radiolabeled TFS-4 will be clinically useful not only for radioimmunodetection but also for targeting radioactive agents in SCLC patients.

\section{REFERENCES}

1) Okabe, T., Kaizu, T., Fujisawa, M., Watanabe, J., Kojima, K., Yamashita, T., and Takaku, F. Monoclonal antibodies to surface antigens of small cell carcinoma of the lung. Cancer Res. 44: 5273-5278, 1984.

2) Okabe, T., Kaizu, T., Fujisawa, M., Watanabe, J., and Takaku, F. Clinical application of monoclonal antibodies to small cell lung cancer. Jpn. J. Med. 24: 250-256, 1985.

3) Watanabe, J., Okabe, T., Fujisawa, M., Takaku, F., Hirohashi, S., and Shimosato, Y. Monoclonal antibod that distinguishes small-cell lung cancer from nonsmall-cell lung cancer. Cancer Res. 47: 826-829, 1987.

4) Okabe, T., and Takaku, F. Diagnostic and therapeutic applications of monoclonal antibodies to small cell carcinoma of the lung. Jpn. J. Clin. Oncol. 16: 243-252, 1986.

\title{
II. Treatment of Cancer
}

\section{Recent Cancer Chemotherapy}

\author{
Kazuo OTA
}

Aichi Cancer Center Hospital, Nagoya

\section{Curative cancer chemotherapy}

Recent progress in cancer chemotherapy is a curative chemotherapy in some malignancies. More than $50 \%$ of child acute lymphatic leukemia patients are curable with chemotherapy. In audlt acute non-lymphocytic leukemia more than $60 \%$ of patients achieved a complete remission and a long term survival rate of $12 \%$ was achieved (Fig. 1). In adult malignant lymphoma patients a survival rate has been markedly improved during the last 20 years. The recent 3 -year survival rate was $75 \%$ in total, around $95 \%$ in clinical stage 1 and 2 and around 60\% even in stage 3 and 4 (Fig. 2).

In solid tumors the benefit of chemotherapy is significant in testicular tumor, ovarian cancer and small cell lung cancer of limited disease. In advanced non-seminomatous testicular tumor 50$70 \%$ of patients achieved a complete remission and $65 \%$ of long term survival rate was achieved with combination chemotherapy including cisplatinum, and $80 \%$ of patients were curable with a salvage therapy of multidisciplinary treatment. Ovarian cancer had been very poorly prognostic, however recent chemotherapy of ovarian cancer with cisplatinum has shown a significant efficacy, and $50 \%$ of the patients with stage III were curable with surgical adjuvant chemotherapy and a second look operation with salvage therapy. 